\title{
Noninvasive Continuous Vagal-Nerve Monitoring, Harnessing the Primitive Laryngeal Adductor Reflex, Is on the Horizon
}

\section{Lisa A. Orloff}

Division of Head and Neck Surgery, Department of Otolaryngology, Stanford University School of Medicine, Palo Alto, California, U.S.A.

Review of: Sinclair CF, Téllez MJ, Ulkatan S 2018 Noninvasive, tube-based, continuous vagal nerve monitoring using the laryngeal adductor reflex: feasibility study of 134 nerves at risk. Head Neck 40:24982506. PMID: 30120890.

\section{SUMMARY}

\section{Background}

Recurrent laryngeal nerve injury is one of the most significant and common complications of thyroid surgery, with rates approaching 10\% when preoperative and postoperative objective laryngeal examinations are performed (1,2). In an effort to reduce injuries, intraoperative nerve monitoring (IONM) during thyroid and related surgeries has grown steadily in popularity and refinement. Noninvasive techniques that use endotracheal tube-based surface electromyographic (EMG) electrodes are preferred. Intermittent IONM (I-IONM) is most common; continuous IONM (C-IONM) currently requires added dissection to place an electrode on the vagus nerve, and is thus more invasive, yet it can provide feedback regarding impending nerve injury that enables surgeons to modify their technique in real-time. In this study (3), Sinclair and colleagues report on an elegant new method of C-IONM that is noninvasive, endotracheal tube-based, and incorporates sensory stimulation to the larynx during general anesthesia to trigger a detectable motor response as part of the innate laryngeal adductor reflex (LAR).

\section{Methods}

Patients undergoing thyroid and parathyroid surgery performed by a single surgeon were monitored by
LAR-C-IONM, in addition to conventional I-IONM. Electrical stimulation with a median current of 8 $\mathrm{mA}$, a duration of 0.1 to $1 \mathrm{msec}$, and a rate of 4 $\mathrm{Hz}$ was delivered through the endotracheal tube to the contralateral laryngeal mucosa, while vocalfold adduction (amplitude of EMG response, in microvolts) was recorded from the operative side. "Opening" and "Closing" LAR values (change in mean EMG amplitude) were correlated with preoperative and postoperative laryngoscopy and Voice Handicap Index (VHI-10) questionnaire results.

\section{Results}

A total of 100 patients with 134 nerves at risk were studied. LAR was elicited in $100 \%$. Mean closing LAR amplitudes were significantly (at least 60\%) lower in patients with abnormal postoperative laryngeal function than in those with normal function (mean [ \pm SD], 93.1 $\pm 47.0 \mu \mathrm{V}$ vs. $270.3 \pm 159.3 \mu \mathrm{V}$ ) and corresponded with significant decreases in VHI-10 scores. The percent decrement was more relevant than the absolute value of LAR amplitude. Of note, for every thyroid surgery in which transient decreases in LAR amplitude occurred without increases in reflex latency, LAR amplitude recovered on release of traction on the recurrent laryngeal nerve during surgery. 


\section{Monitoring, Harnessing the Primitive Laryngeal Adductor} Reflex, Is on the Horizon

\section{Conclusions}

Harnessing the LAR to enable noninvasive C-IONM shows great promise for aiding in the prevention of recurrent laryngeal nerve injuries during thyroid and parathyroid surgeries. The technique and equipment are evolving and will be applicable even to other head and neck and neurosurgical procedures in which the recurrent laryngeal nerve is at risk.

\section{COMMENTARY}

The only currently available technique for continuous vagal nerve monitoring (C-IONM) in thyroid surgery requires exposure of and placement of an electrode around the vagus nerve within the carotid sheath (4). In contrast, the LAR-based C-IONM technique depends solely on endotracheal tubebased electrodes that stimulate afferent and record efferent responses within the vagal reflex arc that comprises the sensory, motor, and central components of the LAR (5). The LAR has been found to be preserved even during total intravenous general anesthesia. The LAR is exquisitely sensitive to changes in traction and stretch on the recurrent laryngeal nerve, which alter efferent neuron excitability. This sensitivity results in signal changes that enable real-time adjustments by the surgeon who is exerting the traction, to relax tension when excessive to avoid injury to the nerve. Any surgeon who instructs others in thyroid surgery knows that one of the most difficult things to judge and to teach trainees is how much traction is too much.

The amplitude of the LAR response is critically dependent on adequate contact of the tube-based electrodes with the laryngeal mucosa. No commercially available endotracheal tube is designed for LAR-C-IONM, so electrode contact by standard tubes may be suboptimal. A tube design with electrodes specifically positioned to optimize stimulating mucosa as well as recording vocal-fold muscle contraction will undoubtedly facilitate adoption of the LAR-C-IONM technique. So will development of systems for "single-surgeon" functionality that eliminate the need for analysis by a neurophysiologist in real-time.

In his New Yorker essay entitled, "Personal Best" (6), Atul Gawande (an endocrine surgeon) describes how when we talk about medical progress, people think of technology. But, he writes, "the capabilities of doctors matter every bit as much as the technology. This is true of all professions. What ultimately makes the difference is how well people use technology." In the case of preventing recurrent laryngeal nerve injury during thyroid surgery, I-IONM has become commonplace, and meanwhile, the LAR is a long-recognized primitive human reflex. By combining the two concepts, surgeons, even experienced surgeons, will be better able to prevent traction injuries to the recurrent laryngeal nerves by receiving actionable feedback from modified existing technology to achieve noninvasive LAR-C-IONM. 

Reflex, Is on the Horizon

\section{References}

1. Randolph GW, Kamani D 2006 The importance of preoperative laryngoscopy in patients undergoing thyroidectomy: voice, vocal cord function, and the preoperative detection of invasive thyroid malignancy. Surgery 139:357-362.

2. Chandrasekhar SS, Randolph GW, Seidman MD, et al 2013 Clinical practice guideline: improving voice outcomes after thyroid surgery. Otolaryngol Head Neck Surg 148(6 Suppl):S1-S37.

3. Sinclair CF, Téllez MJ, Ulkatan S 2018 Noninvasive, tube-based, continuous vagal nerve monitoring using the laryngeal adductor reflex: feasibility study of 134 nerves at risk. Head Neck 40:2498-2506.
4. Schneider R, Randolph GW, Sekulla C, Phelan E, Thanh PN, Bucher M, et al 2013 Continuous intraoperative vagus nerve stimulation for identification of imminent recurrent laryngeal nerve injury. Head Neck 35:1591-1598.

5. Sinclair CF, Tellez MJ, Tapia OR, Ulkatan S, Deletis V 2017 A Novel methodology for assessing laryngeal and vagus nerve integrity in patients under general anesthesia. Clin Neurophysiol. 128:1399-1405.

6. Gawande A 2011 Personal best. The New Yorker. https://www.newyorker.com/ magazine/2011/10/03/personal-best. 